Editorial

\title{
Special Issue of the 2012 conference of the EURO working group Operational Research Applied To Health Services (ORAHS)
}

ORAHS is the European working group on Operational Research Applied to Health Services. It was formed in 1975 as part of a programme for developing special interest groups within EURO, the European branch of the International Federation of Operational Research Societies, IFORS. The focal area of ORAHS' ca. 250 members perfectly aligns with that of the Operations Research for Health Care (ORHC) journal. This special issue of ORHC is dedicated to the 38th annual meeting of the ORAHS working group, from July 15-20 2012, which took place at University of Twente in the Netherlands, and was organized by the university's research centre on the optimization of healthcare processes CHOIR (http://www.utwente.nl/choir). The organizers of this conference, and authors of this editorial, are also the guest editors for this special issue of ORHC.

The conference welcomed 149 participants, and 100 papers were presented on diverse topics regarding the application of $\mathrm{OR} / \mathrm{OM}$ to health care. The theme of the conference was "High Tech - Human Touch": we have challenged the ORAHS community to share their ideas about how OR can contribute to the challenges and opportunities that new technologies bring to the health care sector, and how OR research can lead to an improved working environment for employees and better quality of care. Finally, the implementation of the developed solutions were discussed and the human factor that is involved in this.

Amongst the submitted papers we selected five papers with a diverse range of topics that demonstrate the wide range of possible application of OR for healthcare. A sixth paper is still under review and may appear in a future edition of ORHC. Three papers deal with operations management topics, one paper with treatment optimization, and one is a discussion on the ethical/human dimension of operations research applied to health care. Below, we give a one paragraph description of each contribution.

The first paper in this issue addresses robust resource planning in the context of home care services. There is an increasing pressure on the efficiency and effectiveness of home care services, due to the ageing population and the focus of hospitals on reducing patient length of stay. Lanzarone and Matta study the robust assignment of nurses to patients, under continuity of care. This implies assigning each newly admitted patient to a reference nurse, selected from the eligible nurses in the patient's district. The aim is to minimize the maximum overtime of nurses in a lexicographic sense.

In the second paper, Marques, Captivo, and Pato study the advance and allocation scheduling of elective surgeries using genetic heuristics. They consider two optimization criteria: the operating room utilization and the number of surgeries scheduled. Furthermore, they consider the application of their heuristics to a real hospital in Portugal.

The third paper, the paper of Sharif, Stanford, Taylor and Ziedins, studies the accumulating priority queue (APQ), a priority queue where customer priorities are a function of their waiting time. Examples of APQs can be found in the context of emergency medicine, where different priority classes have different targets for maximum waiting time. In past work, waiting time distributions were derived for when the queue has a single server. This paper provides expressions for the waiting time distributions for a multiserver APQ.

The fourth paper by De Boeck, Beliën and Egyed is a literature review of studies regarding the optimization of doses in brachytherapy. Brachytherapy is an internal radiotherapy where tumors receive a temporary high dose of radiation. A classification of the literature is presented, which reflects the different perspectives of the problem. The paper discusses the shortcomings in the current approaches found in the literature, and provides ideas and opportunities for future research.

The fifth and final paper by Mullen raises the question "are numbers still killing people?", in other words, do operations research approaches for healthcare decision making still ignore the ethical "human" dimension? An ethics discussion is provided on whether it is ever permissible to kill someone, and the extent to which recent analyses, policies and proposals address the various concerns is examined. Finally, it warns this journal's research community that "The force of 'high-tech' analysis must not be allowed to eclipse the "human touch'."

As guest editors we would like to take the opportunity to express our gratitude to the various reviewers of this special issue, who did an excellent job analyzing the submitted papers and providing suggestions for improving them. We also thank the editorial board of ORHC for giving us the opportunity to put together this special issue, and for their advices along the way. And last, but not least, we thank the authors for making their valuable contributions, and for considering ORHC as an outlet for their work.

Erwin W. Hans Ingrid M.H. Vliegen 